\title{
ERRATUM
}

\section{A biocompatible condensation reaction for controlled assembly of nanostructures in} living cells

Gaolin Liang, Hongjun Ren and Jianghong Rao

Nature Chemistry 2, 54-60 (2009); published online 17 December 2009; corrected after print 18 January 2010.

In the version of this Article originally published, in Fig. $1 \mathrm{~b}$ the groups labelled $\mathrm{R}^{1}$ and $\mathrm{R}^{2}$ were switched. This has now been corrected in the HTML and PDF versions of the Article. 